\title{
A TWO-PHASE DEBRIS FLOW MODEL WITH BOULDER TRANSPORT
}

\author{
C. MARTINEZ ${ }^{1}$, R. GARCIA-MARTINEZ ${ }^{3} \&$ F. MIRALLES-WILHELM ${ }^{2}$ \\ ${ }^{1}$ Department of Civil and Environmental Engineering, Florida International University. \\ ${ }^{2}$ Florida International University and FLO-2D Software, Inc. \\ ${ }^{3}$ Department of Earth and Environment, Florida International University.
}

\begin{abstract}
We present a quasi three-dimensional numerical model to simulate debris flows that considers a continuum non-Newtonian fluid phase for water and fine sediments, and a non-continuum phase for large particles such as boulders. Particles are treated in a Lagrangian frame of reference using the 3D Discrete Element Method. The fluid phase is implemented in the RiverFLO-2D model, which solves the 2D depth-averaged shallow water equations with the Finite Element Method on a triangular non-structured mesh. The model considers particle-particle and wall-particle collisions, taking into account that particles are immersed in a fluid and subject to gravity, friction and drag forces. Bingham and Cross rheological models are used for the continuum phase providing very stable results, even in the range of very low shear rates. Results show that Bingham formulation proves better able to simulate the stopping of the fluid when the applied shear stresses are low. Comparing numerical results with analytical solutions and data from flume-experiments demonstrates that the model is capable of replicating the motion of large particles moving in the fluid flow. An application to simulate debris flows that occurred in Northern Venezuela in 1999 shows that the model can replicate the main boulder accumulation reported for that event.
\end{abstract}

Keywords: boulder accumulation, debris flow, discrete element method, finite element method, Lagrangian formulation.

\section{INTRODUCTION}

Debris flow is a frequent phenomenon in mountainous regions. It occurs when masses of poorly sorted sediments, rocks, and fine material, agitated and mixed with water, surge down slopes in response to water flow and gravitational attraction. A typical surge of debris flow has a steep front or 'head' with the densest slurry, the highest concentration of boulders, and the greatest depth. A progressively more dilute and shallower tail follows this head.

Reviews presented by Iverson [1], exhaustively describe the physical aspects of debris flow motion and clearly divide previous debris flow research into two distinct categories. The first, based upon the pioneering work of Johnson [2], assumes that debris flow behaves as a viscoplastic continuum. This model describes a single-phase material that remains rigid unless stresses exceed a threshold value: the plastic yield stress. Various rheological models have been proposed, derived from experimental results or from theoretical considerations, such as the Bingham model [3], the Cross model [4], and the quadratic model proposed by O'Brien and Julien [5]. The Bingham plastic model is the most commonly used in practice.

The second approach has focus on the mechanics of granular materials. Based on Bagnold [6], two-phase models have been developed by several authors, such as Takahashi [7] and Iverson [1]. These models explicitly account for solid and fluid volume fractions and mass changes, respectively.

Despite the considerable progress over the past few years, the flow dynamics and internal processes of debris flows are still challenging in many aspects. In particular, there are many factors related to the movement and interaction of individual boulders and coarse sediments that have not been fully addressed in previous works. Asmar et al. [8] introduced the Discrete 
Element Method (DEM) to simulate the motion of solid particles in debris flows. DEM is a numerical method to model dry granular flows where each particle is traced individually in a Lagrangian frame of reference by solving Newton's equation of motion.

This paper describes the development of a quasi three-dimensional model to simulate stony debris flows, considering a continuum fluid phase, and large sediment particles, such as boulders, as a non-continuum phase. Large particles are treated in a Lagrangian frame of reference using DEM, and the fluid phase composed by water and fine sediments is modeled with an Eulerian approach using the depth-averaged Navier-Stokes equations in two dimensions. Bingham and Cross rheological models are used for the continuum phase. Particle's equations of motion are fully three-dimensional. Numerical simulations have been compared with analytical solutions, with data from laboratory experiments, and with a real debris flow event.

\section{GOVERNING EQUATIONS}

The flow domain is divided in computational cells with triangular base and depth $\mathrm{H}$, as shown in Fig. 1.

Assuming non-Newtonian and incompressible fluid phase, the depth averaged continuity and momentum equations in Cartesian coordinates can be written as follows:

$$
\begin{gathered}
\frac{\partial H}{\partial t}+\frac{\partial(\bar{u} H)}{\partial x}+\frac{\partial(\bar{v} H)}{\partial y}=0 \\
\frac{1}{g} \frac{\partial \bar{u}}{\partial t}+\frac{\bar{u}}{g} \frac{\partial \bar{u}}{\partial x}+\frac{\bar{v}}{g} \frac{\partial \bar{u}}{\partial y}+\frac{\partial \eta}{\partial x}+\frac{F_{D x}}{\rho g}+S_{f x}=0 \\
\frac{1}{g} \frac{\partial \bar{v}}{\partial t}+\frac{\bar{u}}{g} \frac{\partial \bar{v}}{\partial x}+\frac{\bar{v}}{g} \frac{\partial \bar{v}}{\partial y}+\frac{\partial \eta}{\partial y}+\frac{F_{D y}}{\rho g}+S_{f y}=0
\end{gathered}
$$

where $H$ is the water depth, $\eta$ is the free-surface elevation, and $\bar{u} \mathrm{u}$ and $\bar{v}$ are the depth averaged velocities in $x$ and $y$ directions, respectively; $g$ is the gravitational acceleration and $\rho$ is fluid density. $F_{D}$ represents the fluid-solid interaction force exerted on the fluid by particles through the fluid drag force, this force is evaluated as:

$$
\mathbf{F}_{D}=\frac{\sum_{i=1}^{n} \mathbf{F}_{F D i}}{\Delta V}
$$

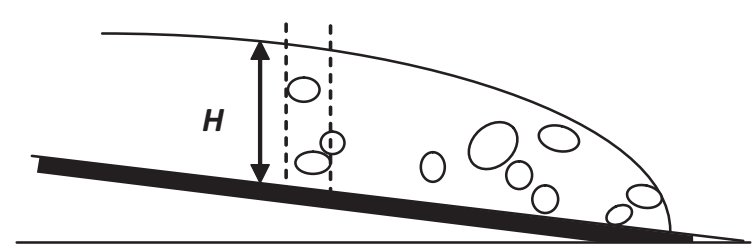

Figure 1: Schematic representation of debris flow with large solid particles. 
Where $\mathbf{F}_{F D i}$ is the fluid drag force on each particle $i, \Delta V$ is the volume of the computational cell, and $n$ is the number of particles in the cell.

$S_{f x}$ and $S_{f y}$ are the depth-integrated stress terms that depend on the rheological formulation used to model the slurry. Assuming a Bingham rheological model and Manning's formula, as proposed by O'Brien and Julien [5], the stress terms for the fluid can be expressed as

$$
\begin{aligned}
& S_{f x}=\frac{\tau y}{\rho g H}+\frac{3 \mu \bar{u}}{\rho g H^{2}}+\frac{N^{2} \bar{u}^{2}}{H^{4 / 3}} \\
& S_{f y}=\frac{\tau y}{\rho g H}+\frac{3 \mu \bar{v}}{\rho g H^{2}}+\frac{N^{2} \bar{v}^{2}}{H^{4 / 3}}
\end{aligned}
$$

Where $N$ is the Manning roughness coefficient.

The fluid dynamic viscosity $\mu$ and yield stress $\tau y$, are determined as functions of the volume sediment concentration $C v$, using the relationships proposed by O'Brien and Julien [9]:

$$
\begin{gathered}
\mu=a_{1} e^{\beta_{1} C v} \\
\tau_{y}=a_{2} e^{\beta_{2} C v}
\end{gathered}
$$

in which $\alpha_{1}, \alpha_{2}, \beta_{1}$ and $\beta_{2}$ are empirical coefficients obtained by data correlation in a number of experiments with various sediment mixtures.

Using a quadratic formulation combined with the Cross rheological model, the stress terms for the fluid are expressed as

$$
\begin{aligned}
& S_{f x}=\frac{\mu_{e f f} \dot{\gamma}}{\rho g H}+\frac{N^{2} \bar{u}^{2}}{H^{4 / 3}} \text { with } \dot{\gamma}=\frac{3 \bar{u}}{H} \\
& S_{f y}=\frac{\mu_{e f f} \dot{\gamma}}{\rho g H}+\frac{N^{2} \bar{v}^{2}}{H^{4 / 3}} \text { with } \dot{\gamma}=\frac{3 \bar{v}}{H}
\end{aligned}
$$

Where $\mu_{\text {eff }}$ is the effective viscosity of the fluid defined by:

with $K_{B}=\frac{\mu_{0}}{\tau_{y}}, \mu_{\infty}=\mu$ and $\mu_{0}=10^{0} \mu$.

$$
\mu_{\text {eff }}=\frac{\mu_{0}+\mu_{\infty} K_{B} \dot{\gamma}}{1+K_{B} \dot{\gamma}}
$$

In the solid phase, spherical particles of different diameters are considered. Particle trajectories are tracked using Newton's second law; considering gravity, buoyancy, fluid drag, and collision forces.

$$
m_{i} \frac{d \mathbf{v}}{d t}=\sum \mathbf{F}_{E}+\sum \mathbf{F}_{N}+\sum \mathbf{F}_{T}
$$


The external force $\mathbf{F}_{E}$ is given by

$$
\mathbf{F}_{E}=\mathbf{F}_{B}+\mathbf{F}_{F D}
$$

The expression to compute the net force acting on the particle due to gravitational effects is

$$
\mathbf{F}_{B}=\frac{4}{3} \pi R^{3}\left(\rho-\rho_{p}\right) \mathbf{g}
$$

Where $R$ is the particle radius and $\rho_{p}$ is the particle density.

The expression for the drag on particles in viscous fluid is given by

$$
\mathbf{F}_{F D}=\frac{1}{2} \pi R^{2} C_{d} \rho|\mathbf{u}-\mathbf{v}|(\mathbf{u}-\mathbf{v})
$$

Where $C_{d}$ is the drag coefficient, $\mathbf{u}$ is the fluid velocity vector at the particle location, and $\mathbf{v}$ is the particle velocity vector.

The last two terms in eqn (12) represent the collision forces or contact forces among particles. Based on the simplified model that uses a spring-dashpot-slider system to represent particle interactions [8], the normal contact force and the tangential contact force are evaluated as

$$
\begin{gathered}
\mathbf{F}_{N}=\mathbf{F}_{N C}+\mathbf{F}_{N D} \\
\mathbf{F}_{T}=\mathbf{F}_{T C}+\mathbf{F}_{T D}
\end{gathered}
$$

The normal contact force $\mathbf{F}_{N C}$ is calculated using a Hook's linear spring relationship,

$$
\mathbf{F}_{N C}=K_{N} \delta_{N}
$$

where $K_{N}$ is the normal contact stiffness and $\delta_{N}$ is the displacement (overlap) between particles $i$ and $j$. The maximum overlap is dependent on the stiffness $K_{N}$. Typically, average overlaps of $0.1-1.0 \%$ are desirable, requiring stiffness of the order $10^{5}-10^{7} \mathrm{~N} / \mathrm{m}$.

The normal damping force $\mathbf{F}_{N D}$ is also calculated using a linear relation given by

$$
\mathbf{F}_{N D}=C_{N} v_{N}
$$

where $v_{N}$ is the normal component of the relative velocity between particles and $C_{N}$ is the normal damping coefficient. This constant $C_{N}$ is chosen to give a required coefficient of restitution $\beta$, defined as the ratio of the normal component of the relative velocities before and after collision.

The tangential contact force, $\mathbf{F}_{T C}$, represents the friction force and it is constrained by the Coulomb friction limit, at which point the particles begin to slide over each other. Prior to sliding, the tangential contact force is calculated using a linear spring relationship,

$$
\mathbf{F}_{T C}=K_{T} \delta_{T}
$$

where $K_{T}$ is the tangential stiffness coefficient, and $\delta_{T}$ is the total tangential displacement between the surfaces of particles $i$ and $j$ since their initial contact. When $K_{T} \delta_{T}$ exceeds the friction limit force $\mu_{f} \mathbf{F}_{N C}$, particle sliding occurs. The sliding condition is defined as

$$
\mathbf{F}_{T C}=\mu_{f} \mathbf{F}_{N C}
$$

where $\mu_{f}$ is the dynamic friction coefficient. 
The tangential damping force $\mathbf{F}_{T D}$ is not included in this model, since it is assumed that once sliding occurs, damping is accounted for from friction. Particle rotation is not considered, since it its impact on boulder transport is assumed to be of much less importance than the friction and drag forces.

Since the fluid phase governing equations are depth-integrated, gradients along $z$ direction are equal to zero in the formulation, as well as the velocity in this direction, $w$. Then, torque in $x$ and $y$ directions are not detectable by the numerical model. Last component is the torque in direction perpendicular to the sloping surface, which is essentially negligible.

Fluid governing eqns (1-3) are solved by the Galerkin Finite Element method using threenode triangular elements. To solve the resulting system of ordinary differential equation, the model applies a four-step time stepping scheme and a selective lumping method, as described by Garcia-Martinez et al. [10].

Forces on each particle are evaluated at each time step, and the acceleration of the particle is computed from the particle governing equation, which is then integrated to find velocity and displacement of each particle.

\section{RESULTS}

\subsection{Analytical solutions}

The first modeling step was the implementation of different rheological models for the simulation of mud flows. This modeling would account for the representation of the fluid phase of the debris flow. The numerical model was run using RiverFLO-2D software, a finite element modeling system for detailed analysis of river hydrodynamics, sediment transport, and bed evolution [10]. In the software, two rheological quadratic formulations were implemented, the first, including Bingham theory and Manning's formula, as proposed by O'Brien and Julien in 1985, and the second, combining Cross formulation and the Manning's formula as proposed in Martinez et al. [11].

To compare with simple results, an analytical solution, proposed by Huang and Garcia [12], was studied and implemented in a computer program. This implementation provided enough data for verification and testing of the new rheological formulations proposed, Martinez et al. [11].

\subsection{Laboratory experiments}

A series of experiments were carried out in a laboratory flume, using homogeneous fine sediment mixtures for the continuum phase and spherical marbles for the discrete phase. The experiments were performed in a $1.9 \mathrm{~m}$ long, $0.19 \mathrm{~m}$ wide, Plexiglas-walled flume, with adjustable slope. The downstream part of the flume was connected to a wood horizontal platform, $0.75 \mathrm{~m}$ long and $0.95 \mathrm{~m}$ wide. A dam-break type of flow was initiated by an abrupt removal of a gate, releasing mixtures from a $0.40 \mathrm{~m}$ long reservoir situated on the upstream part of the flume. Water-clay mixtures were used in all the experiments, with volume sediment concentration $23.5 \%$ and $26.5 \%$. For preparation of the mixtures, kaolinite clay with specific unit weight of 2.77 was used. Fluid density was measured in the laboratory and rheological parameters $\mu$ and $\tau_{y}$ were determined using eqns (7) and (8) in which parameters are $a_{1}=0.621 \times 10^{-3}, \beta_{1}=17.3, \alpha_{2}=0.002$ and $\beta_{2}=40.2$. 
Table 1: Rheological properties of experimental fluids.

\begin{tabular}{lccc}
\hline$C v(\%)$ & $\rho\left(\mathrm{Kg} / \mathrm{m}^{3}\right)$ & $\mu(\mathrm{Pa} . \mathrm{s})$ & $\tau_{y}(\mathrm{~Pa})$ \\
\hline 23.5 & 1410 & 0.0362 & 25.34 \\
26.5 & 1460 & 0.0608 & 84.64 \\
\hline
\end{tabular}

\subsubsection{Experiment 1}

In this experiment the flow of a mixture of $23.5 \%$ volume concentration was studied. The flume bottom slope was set to $4^{\circ}$ and the initial volume released was $6.3 \mathrm{~L}$. For $t=3 \mathrm{~s}$, the wave practically stopped flowing as shown in Fig. 2.

The propagation of the wave was recorded for different times $t$ to construct the spreading diagram showed in Fig. 3.

Fig. 3 compares the experimental data with the numerical solution using Bingham rheological formulation. Numerically, the condition of stopping the fluid is not easy to achieve; however, it is possible to appreciate how the maximum velocity in the fluid decreases with time and it becomes very close to zero about the time the fluid must stop. This fact shows that velocity criteria could be used numerically to stop the fluid.

The following two figures compare longitudinal profiles and maximum velocity for two different mesh sizes. As it is depicted in Figs. 4 and 5, a mesh refinement contributes to improve substantially the solution in the advancing front. The wet-dry algorithm proposed by Garcia at al. [13] was implemented to eliminate dry elements from the calculation, which proved effective to obtain a well-behaved interface between dry and wet elements.

However, the model showed that there is a numerical tendency to form a spurious lowdepth front. The fluid front can be reduced decreasing the element size as well as reducing the minimum depth parameter, which makes the distinction between dry and wet elements. Best results were found with a minimum depth tolerance equal to 0.01 times the average fluid depth as shown in Fig. 5.

\subsubsection{Experiment 2}

In this experiment the same mixture as in experiment 1 was used. In this case, the flume bottom slope was set to $9.54^{\circ}$ and the initial volume released was $6.4 \mathrm{~L}$. The objective of this test was to study the spreading of the fluid in the fan and to study particle movement into the fluid.

14 particles, with diameter $2.5 \mathrm{~cm}$ and density $2500 \mathrm{Kg} / \mathrm{m}^{3}$, were placed in two rows over a small piece of wood in the mud reservoir, just behind the gate. By the time the fluid was released, the piece of wood was quickly removed, so the particles could start their movement along the channel with the fluid.

Figure 6a shows the particles resembling the velocity parabolic distribution across the channel at $t=0.5 \mathrm{~s}$. Blue particles represent those particles placed initially in the first row, orange particles are those placed in the second row. In Fig. 6b, it can be noticed how particles in the center tend to move forward to reach the front of the wave, particles in the second row displace particles in the first row to the sides and these are then left behind because of the fluid velocity gradient. By the time the flow reaches the fan, particles move to the sides of the flow as it is shown in Fig. 7.

Figure 7 compares final position of particles obtained experimentally (final position of particles was measured at the lab) with the numerical results. 


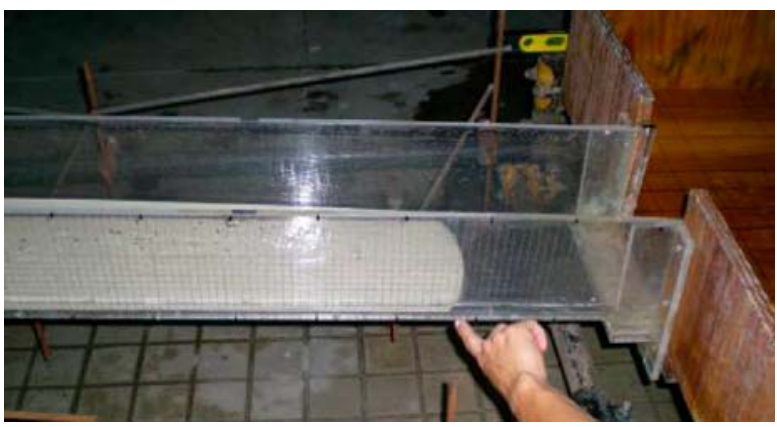

Figure 2: Experiment 1, fluid stops flowing over the sloping channel.

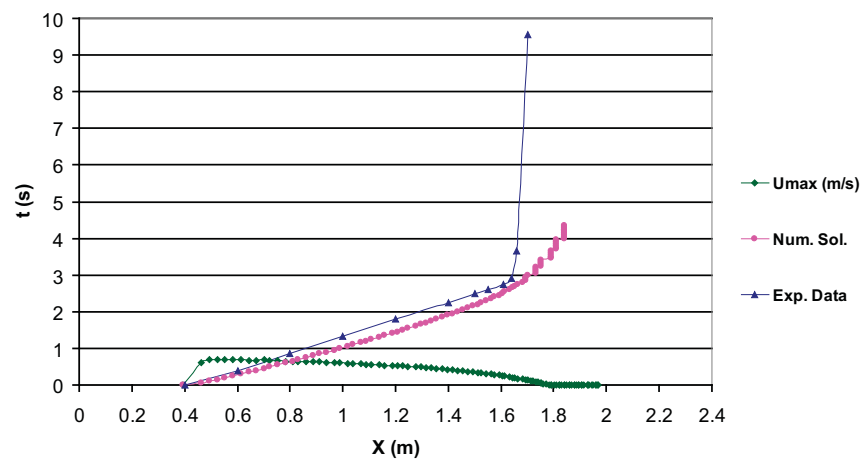

Figure 3: Experiment 1, on the $\mathrm{x}$-axis the distance covered by the flow, on the $\mathrm{y}$-axis the related time. The velocity is also reported, to show its effectiveness as criterion for deciding the fluid stoppage time.

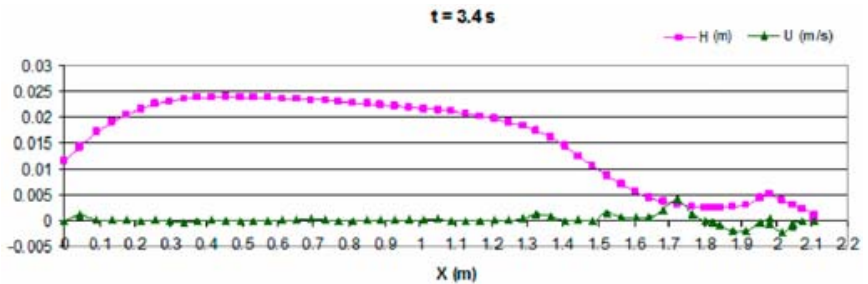

Figure 4: Final free-surface longitudinal profile and $U_{\max }-$ mesh size $0.03 \mathrm{~m}$.

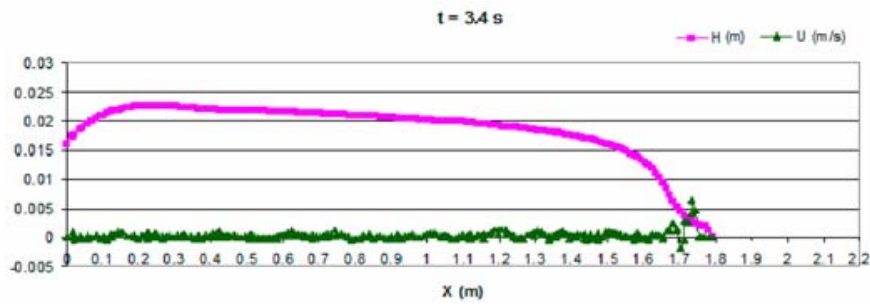

Figure 5: Final free-surface longitudinal profile and $U_{\max }-$ mesh size $0.01 \mathrm{~m}$. 


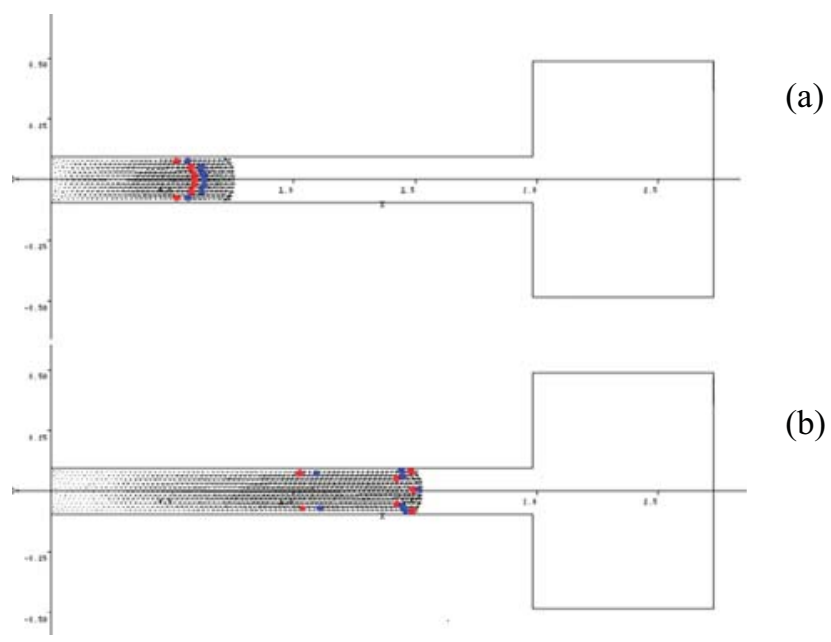

Figure 6: Experiment 2, numerical simulation, (a) above $\mathrm{t}=0.5 \mathrm{~s}$, (b) below $\mathrm{t}=1.6 \mathrm{~s}$.

(a)

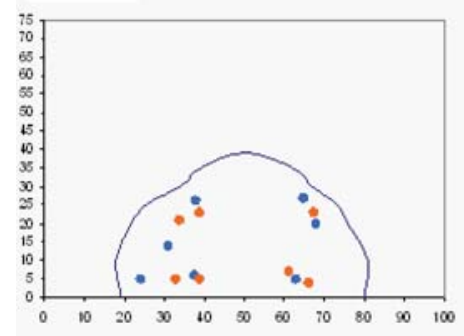

(b)

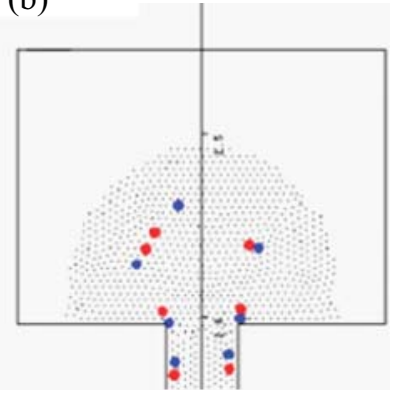

Figure 7: Experiment 2, final position of particles, (a), left experimental data, (b) right numerical solution.

In the numerical solution, it can be seen that there exist some delay on the particles positioned close to the walls; this is due to the velocity boundary condition at the walls. In practical applications of the model, it is necessary to allow slip at the walls, since the no-slip condition formulated in finite elements becomes very restrictive. However, a total slip condition is not possible, since for this case no velocity profile would be created across the channel. In this simulation, $90 \%$ of slip at the wall was considered.

\subsubsection{Experiment 3}

In this experiment, a mixture of volumetric concentration of $26.5 \%$ was studied. In this case, the flume bottom slope was increased to $10.7^{\circ}$ and the initial volume released was $11.1 \mathrm{~L}$. The objective of this test was to study the spreading of the fluid and study particle movement into a mixture with higher clay concentration.

Figure 8 shows the spreading relation in the longitudinal direction for this experiment. This relation is compared with numerical results obtained using Bingham and Cross rheological models. 


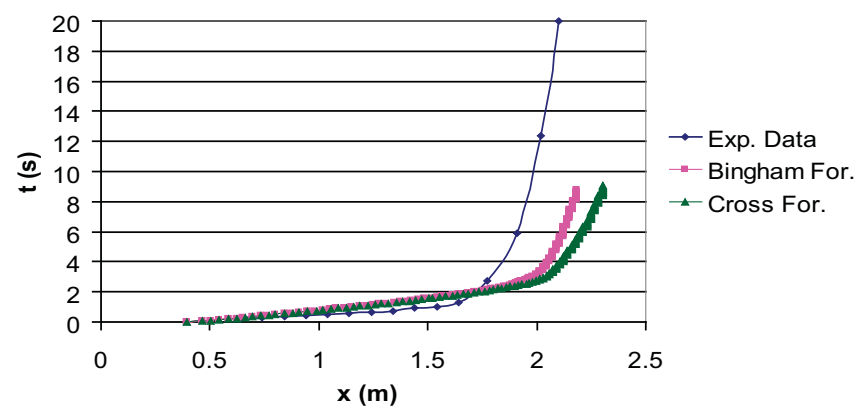

Figure 8: Experiment 3, on the x-axis the distance covered by the flow, on the y-axis the related time. Results carried out using two different rheologies are reported.

Both rheological formulations produce very similar results, but they are not capable of replicating the frontal wave progress for intermediate times; however, they show realistic final fluid coverage, when velocities in the fluid become very close to zero. Bingham formulation demonstrates to be more effective in decreasing the velocities to zero along the fluid edge.

In this experiment 14 particles were placed on the fluid in a similar manner as was done in the previous experiment. Figure 9 compares the final particle positions obtained numerically against final observed particle location. Note that some particles lag behind close to the flume wall and that the general location of the particles on the alluvial fan is very close to the observed locations.

\subsection{Application: Venezuela's 1999 alluvial fan debris flooding event}

Heavy rainfall from a storm on December 14-16, 1999 triggered thousands of shallow landslides on steep slopes of Cerro El Avila, north of Caracas, Venezuela, and caused flooding and massive debris flows in the channels of major drainages that severely damaged coastal communities along the Caribbean Sea. The largest fan on this area is that of San Julián River at Caraballeda, shown in Fig. 10. This fan was one of the most heavily damaged areas in the event. The thickness of sediment deposition, maximum size of transported boulders, and size of inundated area were all notably larger in this drainage in comparison to the other close watersheds.

The U.S. Geological Survey studied the affected area [14], measuring slope, deposit thickness, and boulder size from the fan apex to the distal end of the fan near the coastline. Data was used to map the distribution and thickness of deposits and to draw contours of maximum boulder size, as shown in Fig. 11.

The numerical simulation was performed using a finite element mesh with 22,500 triangular elements. The element characteristic size was $12 \mathrm{~m}$ on average. The topography data used to define the finite element mesh was interpolated from the original cartographic information prior to the event [15].

A 500 year-return period hydrograph was used as flow input at the fan apex, as shown in Fig. 12, with an average volume sediment concentration of $\mathrm{C} v=0.3$. The Manning coefficient considered was equal to 0.065 in the whole fan area, to take into account terrain irregularities. The same value was used by Garcia [15], and was found to be a good estimate for the area. 
(a)

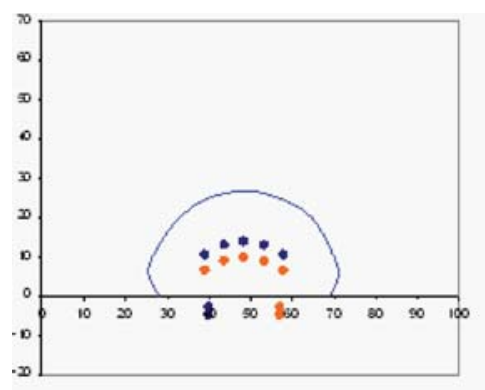

(b)

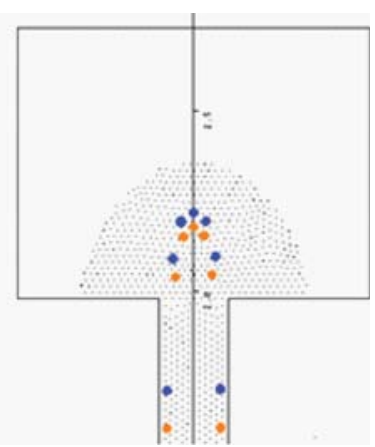

Figure 9: Experiment 3, final position of particles, (a) left experimental data, (b) right numerical solution.

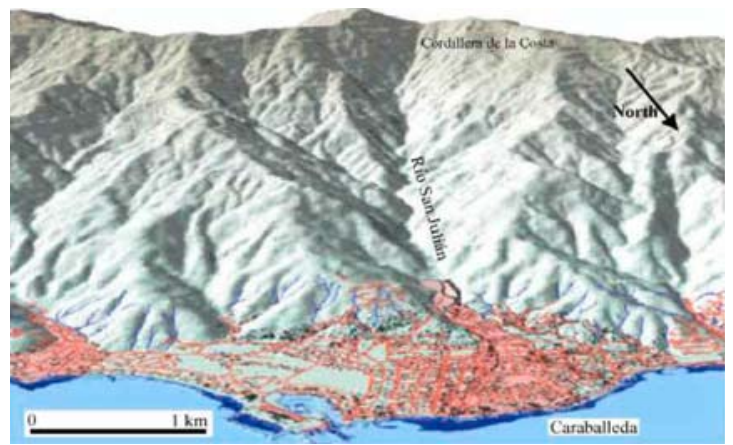

Figure 10: Caraballeda alluvial fan, Venezuela.

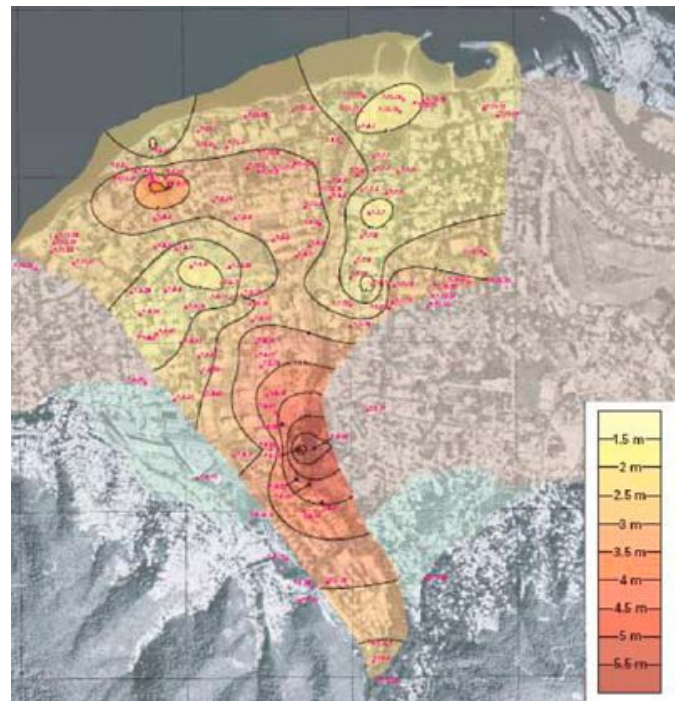

Figure 11: Contours of maximum transported boulder size on Caraballeda alluvial fan, Venezuela. From USGS, 2002. 


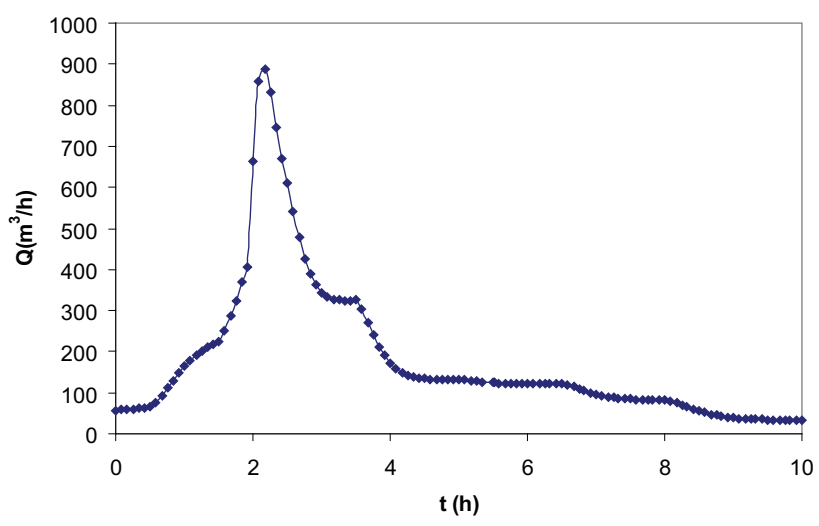

Figure 12: Inflow hydrograph for a 500 year-return period, including solid concentration.

The empirical relationships (7) and (8) were selected for the calculation of fluid rheological properties, using the parameters for water-clay mixtures. As a result of the volume sediment concentration $C v=0.3, \rho=1531 \mathrm{Kg} / \mathrm{m}^{3}, \mu=0.11 \mathrm{~Pa} . \mathrm{s}, \tau_{y}=105 \mathrm{~Pa}$.

During the simulation, 1600 boulders with sizes ranging from $1 \mathrm{~m}$ to $6 \mathrm{~m}$ in diameter were included in the event. The boulders were placed into the fan during the first three hours of simulation, at a rate of 50 particles every $6 \mathrm{~min}$. This amount of particles was selected to ensure a manageable computational time. Density for the boulders is $\rho_{p}=2600 \mathrm{Kg} / \mathrm{m}^{3}$, equal to the density of Gneiss boulders, the type of boulders mostly found in the area by the USGS.

Figure 13 shows the flooded area at time $t=2.2 \mathrm{~h}$, the time corresponding with the peak discharge in Fig. 12. Comparing this region with the post-event aerial view shown in the background, it can be noticed that the model acceptably reproduces the extent of the area affected by the debris flow.

Figure 14 shows the velocity field at time $t=2.2 \mathrm{~h}$. It can be seen that major velocities occurs in the fan apex, where the discharge of the river is simulated. Velocities decrease at the urban areas, ranging from 1.0 to $6 \mathrm{~m} / \mathrm{s}$ at the time of the hydrograph maximum value. Higher velocities are developed in the avulsion zone, at the center of the fan, reaching $10 \mathrm{~m} / \mathrm{s}$. The velocities calculated by the model are in good agreement with those estimated by USGS, which ranged from 1.3 to $13.6 \mathrm{~m} / \mathrm{s}$.

Figure 15a shows how solid particles, boulders, are transported by the flow along the main drainages at time $\mathrm{t}=1.8 \mathrm{~h}$.

It is interesting to see how the largest boulders follow the path of the original concrete channel, at the right side of the fan, while smaller boulders take the central path. According to the USGS report, the slope at the center was $4.0^{\circ}$, while the concrete channel direction, was steeper, with a slope gradient of $5.5^{\circ}$ to $6^{\circ}$, then larger boulders were transported to this side. These values of mean nominal diameter and slope steepness reflect USGS observations that for the larger transported and deposited boulders there was a proportional relationship between boulder size and slope steepness.

Figure $15 \mathrm{~b}$ shows boulder positions after 6 hours of simulation. Smaller boulders continue taking the central direction alignment, some of them reached the shoreline or entered into the sea. Larger boulders were deposited in the avulsion zone or took right direction to the concrete channel. None of these large boulders reached the shoreline. In Fig. 15b, it can be seen that the model predicts reasonably boulder final locations as compared with the field data given in Fig. 11. 


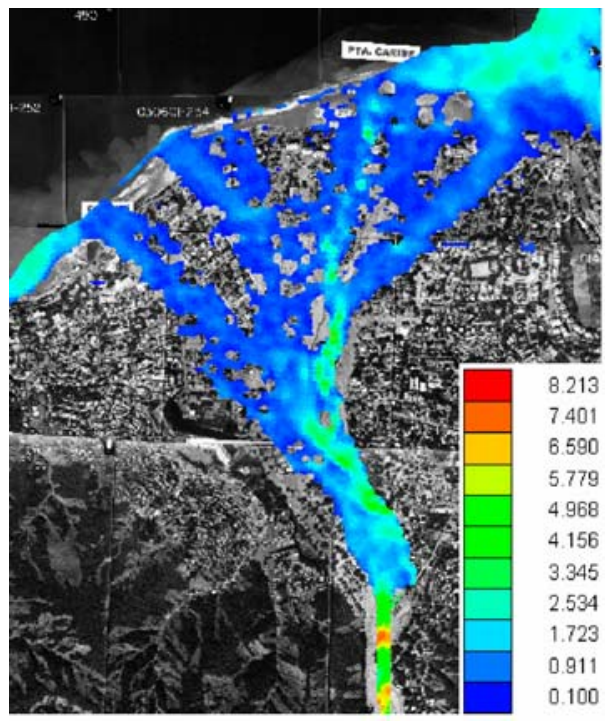

Figure 13: Flooded area at time $\mathrm{t}=2.2 \mathrm{~h}$. Legend indicates flow depth in $\mathrm{m}$.

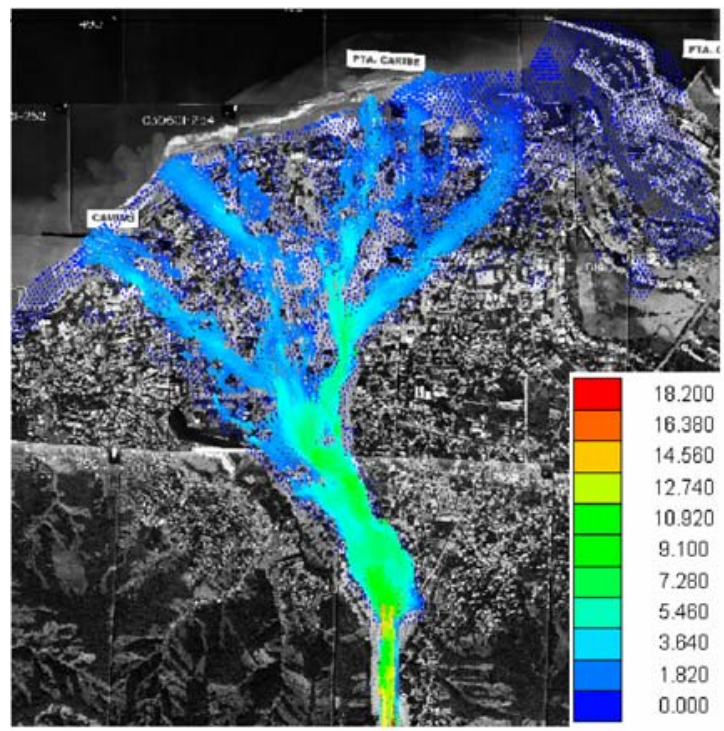

Figure 14: Velocity field at $\mathrm{t}=2.2 \mathrm{~h}$. Legend indicates velocity in $\mathrm{m} / \mathrm{s}$.

According to USGS, the largest boulders were found in the avulsion zone, within a thick matrix, evidence that strongly supports transport by debris flow. At other sites, the largest boulders were observed isolated along the concrete channel, fact that suggests that these boulders moved sliding along the bottom of the channel in a dilute fluid until deposition occurred, USGS Report [14]. 

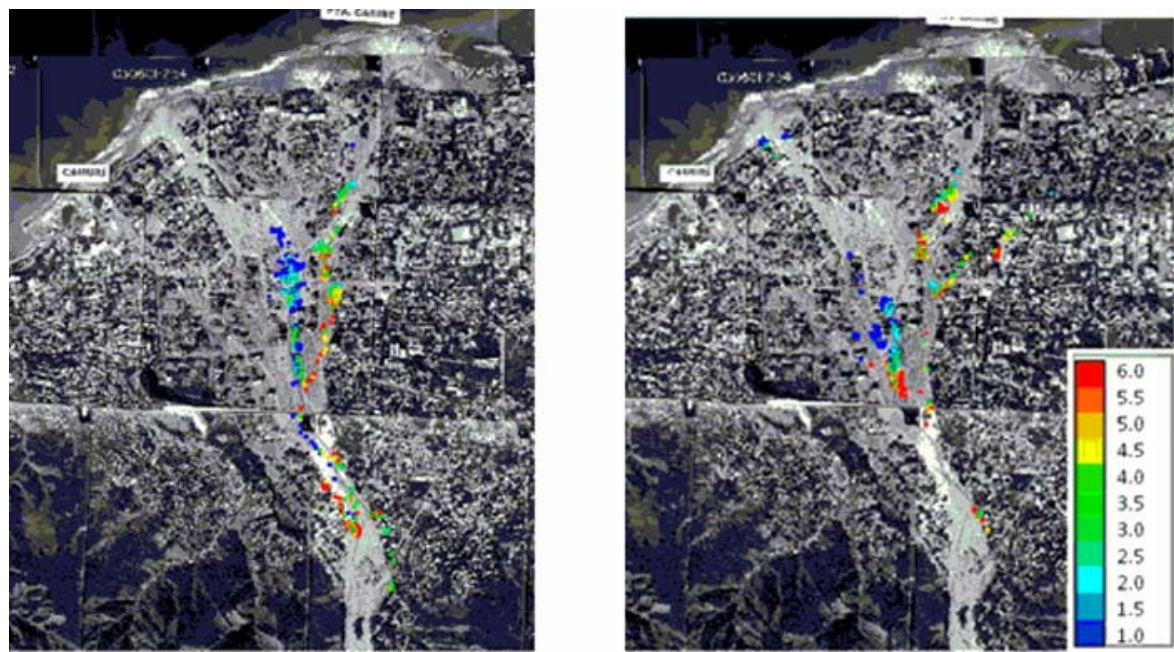

Figure 15: Boulder positions at time (a) $\mathrm{t}=1.8 \mathrm{~h}$, (b) $\mathrm{t}=6.0 \mathrm{~h}$. Legend indicates particle diameter in $\mathrm{m}$.

There is no evidence of big boulders close to the shoreline at this site of the fan.

According to Takahashi [7], during the process of deposition, debris flows deposit the boulders in order from bigger to smaller as it proceeds downstream on alluvial fans. This process was better observed along the central direction and it was also replicated in the numerical simulation.

\section{CONCLUSIONS}

This paper describes the development and application of a quasi three-dimensional two-phase model to simulate debris flows, considering large sediment particles, such as boulders. The continuum non-Newtonian phase is solved by RiverFLO-2D finite element model in two horizontal directions and the particle transport with the Discrete Element Method in 3D.

The model is able to replicate fluid and particle transport when compared against several experiments in a laboratory flume-fan, including the effect of particle-particle and wall-particle collisions.

Bingham and Cross rheological formulations provide very stable results, even when the flow is on the range of very low shear rates. For mud dam-break problems, the Bingham formulation is better able to simulate the stopping stage of the fluid; however, Cross formulation is more accurate for early stages of the solution, where Bingham is not as accurate.

An application to the well-documented debris flow event that occurred in Venezuela in 1999 illustrates the capability of the model to reproduce large-scale real events. Results show that the model reasonably approximates the flood extent affected by the debris flow and the observed boulder accumulation areas, including distribution boulders sizes.

\section{REFERENCES}

[1] Iverson, R.M., The physics of debris flows, Rev. of Geophysics, 35, pp. 245-296, 1997. doi:http://dx.doi.org/10.1029/97RG00426

[2] Johnson, A.M., A Model for Debris flow, Ph.D. dissertation. Pennsylvania State University: University Park, 1965. 
[3] Bingham, E.C. \& Green, H., Paint a plastic material and not a viscous liquid; the measurement of its mobility and yield value. Proceedings of American Society of Testing Materials, 19, pp. 640-664, 1919.

[4] Barnes, H.A., Hutton J.F. \& Walters, K., An introduction to rheology, Elsevier: Amsterdam, 1989.

[5] O'Brien, J.S. \& Julien, P.Y., Physical properties and mechanics of hyperconcentrated sediment flows. ASCE Specialty Conference on the Delineation of Landslides, Debris Flows Hazards, pp. 260-279, 1985.

[6] Bagnold, R.A., Experiments on a gravity-free dispersion of large solid spheres in a Newtonian fluid under shear. Proceedings of the Royal Society of London, 225, pp. 49-63, 1954. doi:http://dx.doi.org/10.1098/rspa.1954.0186

[7] Takahashi, T., Debris Flows, Balkema: Rotterdam, 1991.

[8] Asmar B.N., Langston, P.A. \& Ergenzinger, P., The potential of the discrete element method to simulate debris flow. Debris-Flow Hazards Mitigation: Mechanics, Prediction and Assessment, 1, pp. 435-445, 2003.

[9] O'Brien, J.S. \& Julien, P.Y., Laboratory analysis of mudflows properties. Journal of Hydraulic Eng, 114(8), pp. 877-887, 1988. doi:http://dx.doi.org/10.1061/ (ASCE)0733-9429(1988)114:8(877)

[10] García-Martínez, R., Espinoza, R., Valera, E. \& González, M., An explicit two-dimensional finite element model to simulate short and long term bed evolution in alluvial rivers. Journal of Hydraulic Research, 44(6), pp. 755-766, 2006. doi:http://dx.doi.org/ 10.1080/00221686.2006.9521726

[11] Martinez, C., Miralles-Wilhelm, F. \& Garcia-Martinez, R., A 2D finite element debris flow model based on the cross rheology formulation. Fourth International Conference on Debris-Flow Hazards Mitigation: Mechanics, Prediction and Assessment, Chendu: China, 2007.

[12] Huang, X. \& Garcia, M.H., Asymtotic solution for Bingham debris flows. Debris-flow hazards mitigation: mechanics, prediction and assessment. Proceedings of the First International Conference, ASCE: New York, pp. 561-575, 1991.

[13] Garcia-Martinez, R., Gonzalez, N. \& O'Brien, J., Dam-break flood routing (Chapter 5). Dam-Break Problems, Solutions and Case Studies, ed. D. de Wrachien. \& S. Mambretti, WIT Press, 2009. ISBN: 978-1-84564-142-9.

[14] Wieczorek, G.F., Larsen, M.C., Eaton, L.S., Morgan, B.A. \& Blair, J.L., Debris-flow and flooding hazards associated with the December 1999 storm in coastal Venezuela and strategies for mitigation, U.S. Geological Survey, Open File Report 01-0144, 1991.

[15] Garcia-Martinez, R., Mud Flow Hazard Maps for Vargas State. Final Report for the Avila Project, Fluid Mechanics Institute: University Central of Venezuela (In Spanish), 2008. 